\title{
Long-term Stored Platelets Coupled to Thrombospondin-1 Detection for Rapid and Accurate Identification of Pathogenic HIT Antibodies
}

A Kanack, PhD ${ }^{1 *}$, C Jones, $\mathrm{BS}^{2 *}$, B Singh, $\mathrm{PhD}^{1}, \mathrm{R}$ Leger, $\mathrm{BS}^{1}, \mathrm{NM}$ Heikal, $\mathrm{MD}^{1}$, D Chen, MD, $\mathrm{PhD}^{1}$, RK Pruthi, MBBS ${ }^{3}$, and A Padmanabhan, MBBS PhD ${ }^{1} ¥$

1Department of Laboratory Medicine and Pathology, Mayo Clinic, Rochester, MN, USA

${ }^{2}$ Retham Technologies, Wauwatosa, WI, USA

${ }^{3}$ Department of Medicine, Mayo Clinic, Rochester, MN, USA

* Equal Contribution

$¥$ Corresponding Author

Address for Correspondence:

Anand Padmanabhan, MBBS PhD

Divisions of Hematopathology, Transfusion Medicine and Experimental Pathology

Department of Laboratory Medicine \& Pathology

2001 st Street SW

Mayo Clinic

Rochester, MN 55906

Email: Padmanabhan.Anand@mayo.edu

Abstract Word count: 200

Manuscript Word Count: 1,806

Number of Figures: 2

Number of Tables: 0

Number of References: 22

Number of Supplementary Figures: 3

Conference Presentation: The data were presented in part at the International Society of Thrombosis and Hemostasis Annual Meeting in July 17-21, 2021. 


\section{Abstract}

Heparin-induced thrombocytopenia (HIT) is a potentially life-threatening disorder characterized by antibodies to Platelet Factor 4 (PF4)-polyanion complexes which cause thrombocytopenia and thrombosis. Currently used technically-simple frontline assays such as the PF4-polyanion enzyme-linked immunosorbent assays (ELISAs) lack specificity, and more accurate functional assays such as the serotonin release assay (SRA) and PF4-dependent P-selectin expression assay (PEA) have long turnaround times due to technical complexity and availability only in the reference laboratory setting. There is a critical need for accurate near-patient functional testing to guide patient management, but a key barrier to attaining this goal is the short-term viability of platelets. Here, we detail a process of platelet cryopreservation that renders them viable for at least one year and show that PF4-treated cryopreserved platelets, when coupled with ELISAbased measurement of thrombospondin-1 release (a platelet a-granule protein), detects pathogenic HIT antibodies with high accuracy. Furthermore, testing of a cohort of nonpathogenic HIT antibodies that were strongly reactive in PF4/polyanion ELISA but negative in functional assays demonstrated negative results in the thrombospondin-1 release assay, confirming high specificity of this technique. These findings have the potential to transform the diagnostic testing paradigm in HIT by making in-hospital functional testing available for rapid and accurate diagnosis. 


\section{Introduction}

HIT is a disorder characterized by antibodies to PF4/polyanion complexes that cause thrombocytopenia and thrombosis ${ }^{1-4}$. A recent large epidemiological study suggests high morbidity and a mortality rate of $\sim 10 \%$ in HIT patients ${ }^{5}$. Currently, two classes of assays are used for the HIT diagnosis. PF4/polyanion enzyme-linked immunosorbent assay (ELISA)-based tests are simple from a technical standpoint but are highly non-specific ${ }^{6}$. For example, in a recent $400+$ patient prospective HIT diagnostic study, only $50 \%$ of ELISA-positive samples harbored platelet-activating antibodies ${ }^{7}$. Thus, reliance on these assays results in an "epidemic of overdiagnosis"8 and excessive use of alternative anticoagulants that have a higher risk of bleeding $^{6,9}$. In addition to the medical risks associated with excessive use of non-heparin anticoagulants, they are expensive ${ }^{10}$.

On the other hand, more accurate functional (platelet-activation based) assays such as the serotonin-release assay (SRA) ${ }^{11}$ and heparin-induced platelet activation assay (HIPA) ${ }^{12}$ require complex techniques and handling of radioactive reagents or aggregometry equipment. Thus, functional assays are available only in the context of reference laboratories. Recent studies suggest that the use of PF4-treated platelets in functional HIT testing is equivalent or superior to the use of heparin-treated platelets for HIT diagnosis. ${ }^{7,13,14}$ A new assay developed based on these findings, the PF4-dependent P-selectin expression assay (PEA), although simpler, still requires flow cytometry ${ }^{7}$ and is currently offered by only one laboratory in the United States. Thus, functional testing is associated with long turnaround times, making their use impractical for immediate patient management.

Therefore, there is a critical need to develop rapid and accurate (functional) diagnostic assays for HIT that can be implemented in the near-patient, in-hospital environment. A key challenge that has prevented the development of self-contained functional HIT in vitro diagnostic assays (IVDs) is the extremely short viability of platelets. Here, we detail the development of a process of cryopreservation that extends platelet viability for at least one year. We also demonstrate that PF4-treated cryopreserved platelets can support the detection of pathogenic, platelet-activating HIT antibodies by measuring the release of an abundant platelet $\alpha$-granule protein, thrombospondin-1 (TSP1) in a simple ELISA-based format.

\section{Methods}

Platelet Cryopreservation 
Platelet-rich plasma (PRP) units sourced from whole blood donations anticoagulated with citrate phosphate dextrose from healthy blood donors were obtained two days after draw, upon completion of infectious disease testing. Anticoagulant citrate dextrose-A (1.2mM sodium citrate; $1.4 \mathrm{mM}$ dextrose, $\mathrm{pH}$ 5.9) and prostaglandin E-1 $(50 \mu \mathrm{g} / \mathrm{mL}$ stock, Millipore Sigma, St. Louis, MO) and was added 1:10 (v/v) and 1:1000 (v/v) respectively to the two day old PRP units. The PRP was centrifuged at $100 \mathrm{~g}, 15$ minutes. Supernatant was collected and centrifuged at $1000 \mathrm{~g} 15$ minutes to pellet platelets. Supernatant was discarded, and platelets were resuspended at a concentration of $1 \times 10^{6} / \mu \mathrm{L}$ and incubated at $37^{\circ} \mathrm{C}$ for 2 hours in a buffer containing the cryoprotectant, trehalose (9.5 mM HEPES; $100 \mathrm{mM} \mathrm{NaCl} ; 4.8 \mathrm{mM} \mathrm{KCl} ; 5.0 \mathrm{mM}$ glucose; $12 \mathrm{mM} \mathrm{NaHCO}_{3} ; 50 \mathrm{mM}$ trehalose, $\mathrm{pH}$ 6.8). 30\% (w/v) bovine serum albumin (BSA, Millipore Sigma, St. Louis, MO) was added to a final $4 \%(\mathrm{w} / \mathrm{v})$ concentration. This platelet solution was then aliquoted in $500 \mu \mathrm{L}$ volumes per tube. Platelets were cryopreserved in a Thermo Scientific CryoMed 7450 controlled rate freezer at a range of freezing rates. Platelets cryopreserved at an optimal rate of $4^{\circ} \mathrm{C} / \mathrm{min}$ to the target of $-80^{\circ} \mathrm{C}$ are presented in this study. Platelets were stored at $-80^{\circ} \mathrm{C}$ for various periods before testing, as noted in the Results and Discussion section.

\section{Thrombospondin Release Assay (TRA)}

Thrombospondin-1 (TSP1), a highly expressed protein in platelet $\alpha$-granules ${ }^{15}$ released upon HIT-antibody mediated platelet activation, was measured by ELISA. Briefly, after method optimization (see Supplementary Appendix for initial conditions), cryopreserved platelet tubes were thawed at $37^{\circ} \mathrm{C}$ and centrifuged at $1000 x g$ for 5 minutes. Supernatants were discarded, and platelets were resuspended in a modified Ringer's Citrate Dextrose (RCD) buffer containing trehalose $\left(108 \mathrm{mM} \mathrm{NaCl} ; 3.8 \mathrm{mM} \mathrm{KCl} ; 1.7 \mathrm{mM} \mathrm{NaHCO}_{3} ; 22.9 \mathrm{M}\right.$ sodium citrate; $27.8 \mathrm{mM}$ Glucose; $50 \mathrm{mM}$ trehalose, $\mathrm{pH} 6.5$ ) and centrifuged at 1000xg for 5 minutes. Supernatants were discarded. Platelets were resuspended in $80 \mu \mathrm{L}$ of a phosphate-buffered saline-based (PBS) activation buffer (137mM NaCl; $2.7 \mathrm{mM} \mathrm{KCl} ; 10 \mathrm{mM} \mathrm{Na}{ }_{2} \mathrm{HPO} 4 ; 1.8 \mathrm{mM} \mathrm{KCl} ; 1 \%$ [w/v] Bovine Serum Albumin[BSA]; 50mM trehalose; 150 $\mathrm{g} / \mathrm{mL}$ PF4 [Protein Foundry, Wauwatosa, WI], pH 7.4) and incubated at room temperature for 20 minutes. $20 \mu \mathrm{L}$ of patient serum was added to the PF4-treated platelets, incubated for 30 minutes at room temperature, and supernatants were collected after centrifugation at 1000xg for 5 minutes. In experiments using thrombin receptor activating peptide (TRAP), platelets were prepared similarly but were not treated with PF4. TRAP $(125 \mu \mathrm{g} / \mathrm{mL}$ stock, AnaSpec, Fremont, CA) or buffer control was added in lieu of serum to a final concentration of $25 \mu \mathrm{g} / \mathrm{mL}$. TSP1 was quantified from platelet supernatants using a 
Thrombospondin-1 DuoSet ELISA kit (R\&D Systems, Minneapolis, MN) using manufacturer instructions.

\section{Patient Samples}

Preliminary testing was performed with apheresates from therapeutic plasma exchange procedures from three patients with confirmed HIT. Thirty-four samples, 16 PEA-positive and 18 PEA-negative samples were identified among remnant sera available in adequate volumes after HIT ELISA diagnostic testing at Mayo Clinic (Rochester, MN). Two additional PEA-positive samples used were apheresates from therapeutic plasma exchange procedures on patients with confirmed HIT. All samples had IgG-specific ELISA (Immucor, Norcross, GA) and SRA results available. Studies were approved by the Institutional Review Board of Mayo Clinic.

\section{Results and Discussion}

In initial studies, we evaluated the ability of one-week-old refrigerated $\left(4^{\circ} \mathrm{C}\right)$ platelets to support detection of HIT antibodies in functional testing using PF4-treated platelets, compared to 2-day old platelets obtained from room temperature stored PRP bags (Supplementary Fig S1). Results demonstrated strong baseline platelet activation due to refrigeration, as evidenced by increased P-selectin (CD62p) expression even upon incubation with healthy donor sera (Supplementary Fig S1), suggesting that long-term refrigeration of platelets is not a viable option for platelet storage. Our observation of increased activation with cold-stored platelets agrees with previously published observations that refrigerated platelets undergo structural, metabolic, and functional changes that impact their activation state ${ }^{16,17}$. As an alternative to refrigeration, we explored the possibility of using cryopreserved platelets in functional testing. Cryopreserved platelets, developed as described in Methods, were initially treated with a strong platelet agonist, TRAP, which resulted in robust activation as evidenced by high TSP-1 release (190 $\mu \mathrm{g} / \mathrm{mL}$ vs. $26 \mu \mathrm{g} / \mathrm{mL}$ with buffer control; Supplementary Fig S2).

Next, we assessed the feasibility of using PF4-treated cryopreserved platelets to detect plateletactivating HIT antibodies. Initially, 20 platelet lots, each obtained from a different healthy donor were used in the study (Figure 1A). Platelets were cryopreserved as described, thawed, treated with PF4, and incubated with three platelet-activating HIT or normal control samples to assess their ability to elicit platelet activation in the thrombospondin-1 release assay (TRA). Despite some variability, HIT samples stimulated higher TSP1 release relative to controls for each of the 20 platelet lots tested (Figure 1A). To assess the longer-term viability of cryopreserved platelets 
for HIT antibody detection, the TRA was repeated using seven platelet lots that had been frozen for more than four weeks (Figure 1B), and demonstrated that platelets remained viable and capable of degranulation upon activation even after storage. Of note, the reactivity of platelets at these two time points was highly reproducible within each platelet lot. Together, this data suggests that while all platelets were "reactive" in the TRA, some may be more reactive than others, a phenomenon that has been documented with other HIT functional assays such as the $\mathrm{SRA}^{18}$. Critically, longer-term storage of platelets for 12 months supported HIT antibodymediated, PF4-dependent platelet activation resulting in the release of significant amounts of TSP1 (Figure 1C).

To assess the diagnostic accuracy of the cryopreserved platelet-coupled TRA, a cohort of 18 PEA-positive and 18 PEA-negative HIT-suspected patient samples were selected for the study (Figure 2A). The cohort was specifically selected to include several cases with "false-positive" IgG-specific ELISA results, i.e., ELISA-positive but negative in functional testing. In this cohort, PEA-SRA concordance was demonstrated in 34 of 36 samples (Figure 2A). Two PEA-positive samples were SRA-negative. One of the SRA-negative samples was an apheresate from a confirmed HIT-positive patient (ELISA OD 2.5, and SRA positive at 62\%). The likely reason for negative SRA results with apheresate was sample dilution secondary to apheresis and is in line with published observations of lower analytical sensitivity of the SRA relative to the PEA ${ }^{14}$. The second PEA-positive/SRA-negative sample was from an ELISA-positive patient with thrombosis and thrombocytopenia following heparin exposure who demonstrated recovery of platelet counts upon heparin cessation, suggesting the high likelihood of SRA-negative HIT, an entity that multiple groups have recently described ${ }^{2,13,19}$.

All 18 PEA-positive samples strongly activated cryopreserved platelets, with a mean TSP1 concentration of $147 \mu \mathrm{g} / \mathrm{mL}$ in platelet supernatant. In comparison, the 18 non-activating PEAnegative samples induced a mean TSP1 release of $43 \mu \mathrm{g} / \mathrm{mL}$ and showed clear separation from the PEA-positive group (Figure 2B). Of note, 11 of the 18 patients in the PEA-negative cohort were ELISA-positive (Figure 2B). To stringently assess the specificity of the TRA, PEA-positive and PEA-negative groups matched for strong ELISA positivity (1.0-2.2 OD) from within the 36sample cohort were compared in a subset analysis (Figure 2C). PEA-positive $(n=7)$ and PEAnegative $(n=4)$ groups had a mean ELISA O.D. of 1.88 and 1.67 , respectively $(p=0.30)$. TSP1 released by activating versus non-activating samples were significantly different with no overlap between the two groups, as shown in Figure $\mathbf{2 C}$, demonstrating the high specificity of the cryopreserved platelet-coupled TRA. Of note, studies presented in Figures 2B-C were 
conducted using cryopreserved platelets that were shipped on dry ice for $\sim 30$ hours, demonstrating the viability of platelets with cold chain-compatible transport. Proof-of-concept studies presented in Supplemental Figure S3 also demonstrate that heparin-treated cryopreserved platelets, like PF4-treated platelets, can be used to detect platelet-activating HIT antibodies.

In summary, using a heterogeneous group of patient samples, including those that were strongly false-positive in the ELISA, cryopreserved platelets coupled to a TSP-1 ELISA endpoint demonstrated $100 \%$ sensitivity and specificity for the detection of platelet-activating HIT antibodies. While platelet activatability after long-term storage (12-months) has been demonstrated, studies to evaluate platelet stability at 18 and 24-months are ongoing. The ability to use 2-day old platelets from PRP units, which are available in abundance from the blood banking industry, should facilitate large-scale production of platelets for in vitro diagnostic kit use. Pooling of platelets, a strategy that we have used successfully for a recently deployed functional HIT diagnostic assay, the $\mathrm{PEA}^{7}$, will be employed in this setting to minimize lot variation in platelet activatability. These findings have the potential to transform the diagnostic testing paradigm in HIT by making in-hospital functional testing available for rapid and accurate diagnosis by the deployment of a self-contained HIT IVD assay that will not require the user to obtain donor platelets. Emerging data suggest that anti-PF4 antibodies in Vaccine-induced immune thrombocytopenia and thrombosis (VITT) seen after some COVID-19 vaccines are consistently detected using PF4-treated platelets as opposed to heparin-treated platelets ${ }^{20-22}$. Thus, findings reported here may be relevant for the detection of VITT antibodies, and studies are planned.

\section{Acknowledgments}

We would like to thank Stephanie Hafner, BS from Mayo Clinic's Research Innovation Office for exceptional research coordination support. This work was supported, in part, by National Institutes of Health grant HL147734 (C.J), HL133479 (A.P) and through a grant from the Therapeutic Accelerator Program of the Drug Discovery Center at Medical College of Wisconsin and Wisconsin Economic Development Corporation's (WEDC): Targeted Industry projects.

\section{Authorship}

AK, CJ, BS provided critical input on laboratory and clinical elements of the study. AK and CJ performed the Thrombospondin-1 release assay and flow cytometry studies. RL, NMH, DC, and RKP provided helpful advice on clinical and laboratory aspects of the manuscript. AP conceived 
medRxiv preprint doi: https://doi.org/10.1101/2021.09.01.21262974; this version posted September 5, 2021. The copyright holder for this preprint

(which was not certified by peer review) is the author/funder, who has granted medRxiv a license to display the preprint in perpetuity.

It is made available under a CC-BY-ND 4.0 International license.

the experimental plan and directed the laboratory studies. AK, CJ, and AP wrote the first draft and all authors provided input and approved the final version.

\section{Conflict-of-interest disclosure:}

CJ reports pending/issued patents (Versiti BloodCenter of Wisconsin, Retham Technologies)

and reports equity ownership and employment in Retham Technologies. R.K.P. reports

honoraria for advisory board participation from CSL Behring, Genentech, Bayer Healthcare AG,

HEMA Biologics, Instrumentation Laboratory, and Merck. AP reports pending/issued patents

(Mayo Clinic, Retham Technologies and Versiti BloodCenter of Wisconsin), equity ownership in

Retham Technologies, and serves on the advisory board of Veralox Therapeutics. 


\section{Figure Legends}

Figure 1. Cryopreserved frozen platelets are activated by HIT antibodies. Thrombospondin1 release assays (TRA) was performed with three platelet-activating HIT (closed circles) and control (open circles) samples using (A) Twenty lots of cryopreserved platelets and (B) Seven cryopreserved platelet lots stored for $>4$ weeks at $-80^{\circ} \mathrm{C}$ (" $4 \mathrm{~W}$ ") and compared to activation of platelets stored for $\leq 1$ week at $-80^{\circ} \mathrm{C}$ ("B", baseline). (C) shows PF4-dependent activation induced by one HIT (closed circle) and one normal control sample (open circle) with three cryopreserved platelet lots stored at $-80^{\circ} \mathrm{C}$ for 12 -months. " $4 \mathrm{~W}$ " platelets ranged in storage age from 29 to 36 days, while "B" platelets ranged in storage age from 1 to 7 days. Date shown in (A) and (B) wee obtained using preliminary methods, as described in the supplementary appendix. Y-axis depicts TSP1 concentration (in $\mu \mathrm{g} / \mathrm{mL}$ ) measured in platelet supernatant and $\mathrm{X}$-axis depicts platelet lot tested.

\section{Figure 2. The TRA had high sensitivity and specificity for detection of pathogenic} platelet-activating HIT antibodies. (A) PEA and SRA results of the patient cohort used in the study are shown on the $\mathrm{X}$-axis and $\mathrm{Y}$-axis, respectively. Red dotted lines represent positive cutoff values (35\% p-selectin expression and $20 \%$ serotonin release). The two patient samples highlighted in red tested positive in the PEA, but negative in the SRA, and had case histories consistent with HIT. (B) TRA results are displayed for the patient cohort after qualitatively grouping based on PEA results as positive (circles), or negative (squares). Patients that tested negative in the PEA but had positive IgG-specific HIT ELISA results are displayed as red squares (11 samples). Each data point represents the mean of technical triplicates. TSP-1 concentrations $(\mu \mathrm{g} / \mathrm{mL})$ are depicted on the $Y$-axis with the mean $+/-1$ SD for each group displayed. (C) TRA results of ELISA OD-matched PEA+/SRA+ vs PEA-/SRA-patients are presented. Seven patients had platelet-activating antibodies (closed circles) while four were did not (red squares). Y-axis depicts TSP1 concentration (in $\mu \mathrm{g} / \mathrm{mL}$ ) measured in platelet supernatant and $\mathrm{X}$-axis depicts the ELISA optical density. Each data point represents the mean of technical triplicates. A two-tailed, unpaired Student's $t$ test was used for comparison. ${ }^{* \star \star} \mathrm{p}<$ 0.0001 . 


\section{References}

1. Greinacher A. CLINICAL PRACTICE. Heparin-Induced Thrombocytopenia. N Engl J Med. 2015;373(3):252-261.

2. Warkentin TE. Laboratory diagnosis of heparin-induced thrombocytopenia. Int J Lab Hematol. 2019;41 Suppl 1:15-25.

3. Cuker A. Clinical and laboratory diagnosis of heparin-induced thrombocytopenia: an integrated approach. Semin Thromb Hemost. 2014;40(1):106-114.

4. Arepally GM, Cines DB. Pathogenesis of heparin-induced thrombocytopenia. Trans/ Res. 2020;225:131-140.

5. Dhakal B, Kreuziger LB, Rein L, et al. Disease burden, complication rates, and healthcare costs of heparin-induced thrombocytopenia in the USA: a population-based study. Lancet Haematol. 2018;5(5):e220-e231.

6. Hicks LK, Bering $\mathrm{H}$, Carson KR, et al. Five hematologic tests and treatments to question. Blood. 2014;124(24):3524-3528.

7. Samuelson Bannow B, Warad DM, Jones CG, et al. A prospective, blinded study of a PF4-dependent assay for HIT diagnosis. Blood. 2021;137(8):1082-1089.

8. Cuker A. Heparin-induced thrombocytopenia (HIT) in 2011: an epidemic of overdiagnosis. Thromb Haemost. 2011;106(6):993-994.

9. Pishko AM, Lefler DS, Gimotty $P$, et al. The risk of major bleeding in patients with suspected heparin-induced thrombocytopenia. J Thromb Haemost. 2019;17(11):1956-1965.

10. Caton S, O'Brien E, Pannelay AJ, Cook RG. Assessing the clinical and cost impact of on-demand immunoassay testing for the diagnosis of heparin induced thrombocytopenia.

Thromb Res. 2016;140:155-162.

11. Sheridan D, Carter C, Kelton JG. A diagnostic test for heparin-induced thrombocytopenia. Blood. 1986;67(1):27-30.

12. Greinacher A, Michels I, Kiefel V, Mueller-Eckhardt C. A rapid and sensitive test for diagnosing heparin-associated thrombocytopenia. Thromb Haemost. 1991;66(6):734-736.

13. Vayne C, Guery EA, Kizlik-Masson C, et al. Beneficial effect of exogenous platelet factor 4 for detecting pathogenic heparin-induced thrombocytopenia antibodies. Br J Haematol. 2017;179(5):811-819.

14. Padmanabhan A, Jones CG, Curtis BR, et al. A Novel PF4-Dependent Platelet Activation Assay Identifies Patients Likely to Have Heparin-Induced

Thrombocytopenia/Thrombosis. Chest. 2016;150(3):506-515.

15. Leung LL. Role of thrombospondin in platelet aggregation. J Clin Invest. 1984;74(5):1764-1772.

16. Hoffmeister KM, Felbinger TW, Falet $\mathrm{H}$, et al. The clearance mechanism of chilled blood platelets. Cell. 2003;112(1):87-97.

17. Winokur R, Hartwig JH. Mechanism of shape change in chilled human platelets. Blood. 1995;85(7):1796-1804.

18. Warkentin TE, Hayward CP, Smith CA, Kelly PM, Kelton JG. Determinants of donor platelet variability when testing for heparin-induced thrombocytopenia. J Lab Clin Med. 1992;120(3):371-379.

19. Pandya KA, Johnson EG, Davis GA, Padmanabhan A. Serotonin release assay (SRA)negative HIT, a newly recognized entity: Implications for diagnosis and management. Thromb Res. 2018;172:169-171.

20. George G, Friedman KD, Curtis BR, Lind SE. Successful treatment of thrombotic thrombocytopenia with cerebral sinus venous thrombosis following Ad26.COV2.S vaccination. Am J Hematol. 2021. 
medRxiv preprint doi: https://doi.org/10.1101/2021.09.01.21262974; this version posted September 5, 2021. The copyright holder for this preprint

(which was not certified by peer review) is the author/funder, who has granted medRxiv a license to display the preprint in perpetuity.

It is made available under a CC-BY-ND 4.0 International license.

21. Greinacher A, Thiele T, Warkentin TE, Weisser K, Kyrle PA, Eichinger S. Thrombotic Thrombocytopenia after ChAdOx1 nCov-19 Vaccination. N Engl J Med. 2021;384(22):20922101.

22. Bourguignon A, Arnold DM, Warkentin TE, et al. Adjunct Immune Globulin for VaccineInduced Thrombotic Thrombocytopenia. N Engl J Med. 2021. 
medRxiv preprint doi: https://doi.org/10.1101/2021.09.01.21262974; this version posted September 5, 2021. The copyright holder for this preprint (which was not certified by peer review) is the author/funder, who has granted medRxiv a license to display the preprint in perpetuity.

Figure 1

A

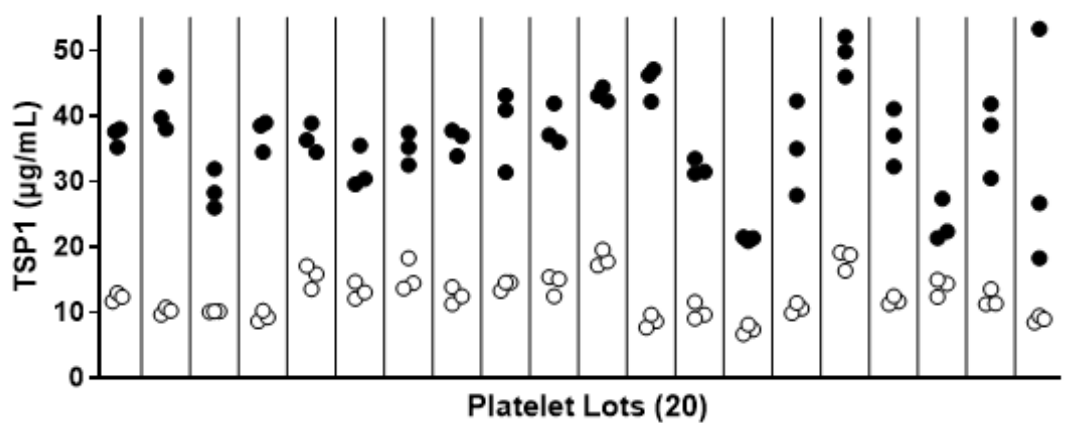

B
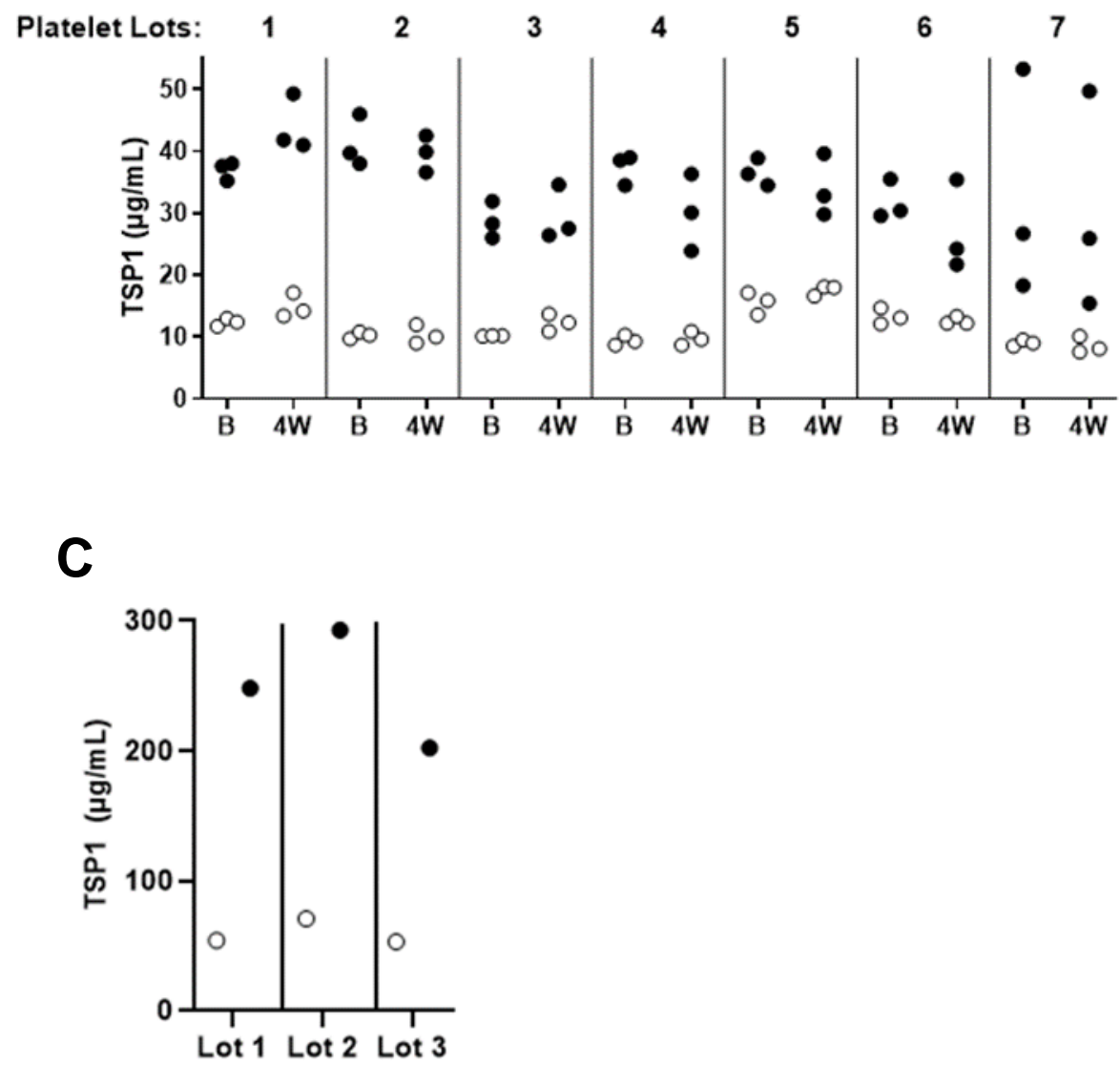
Figure 2

A

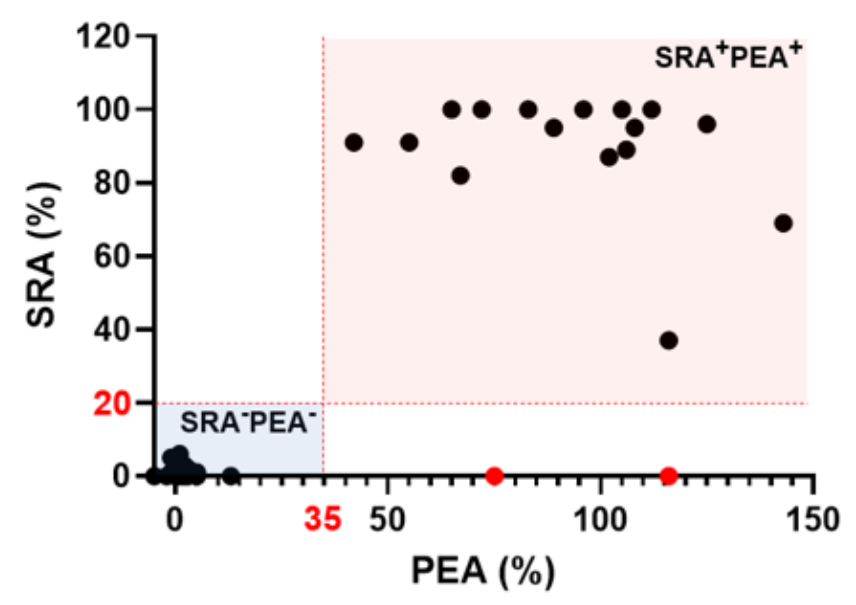

C

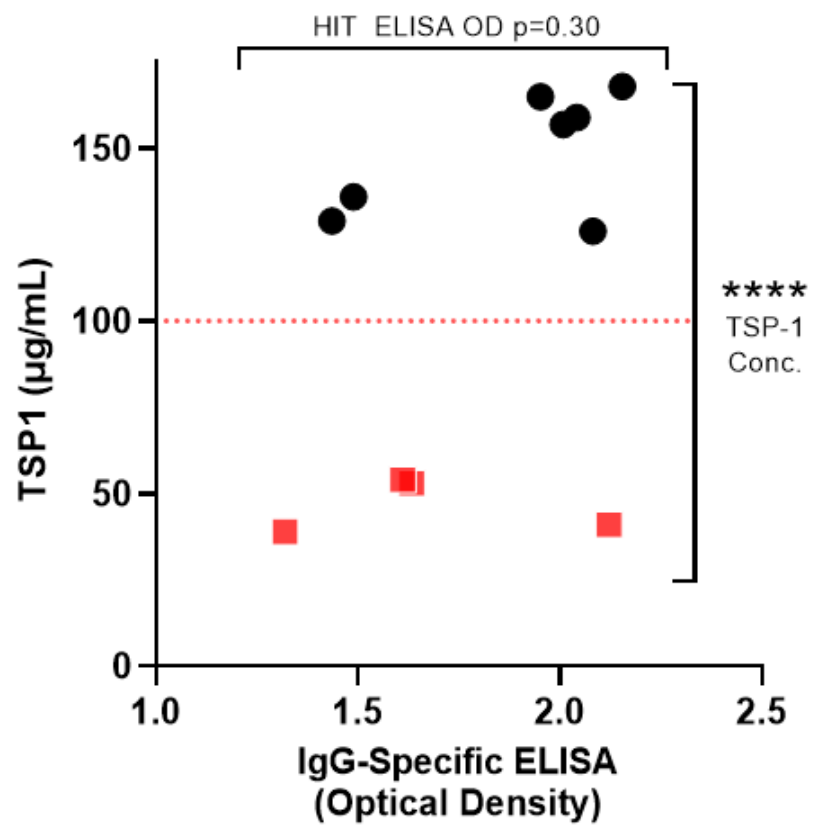

B

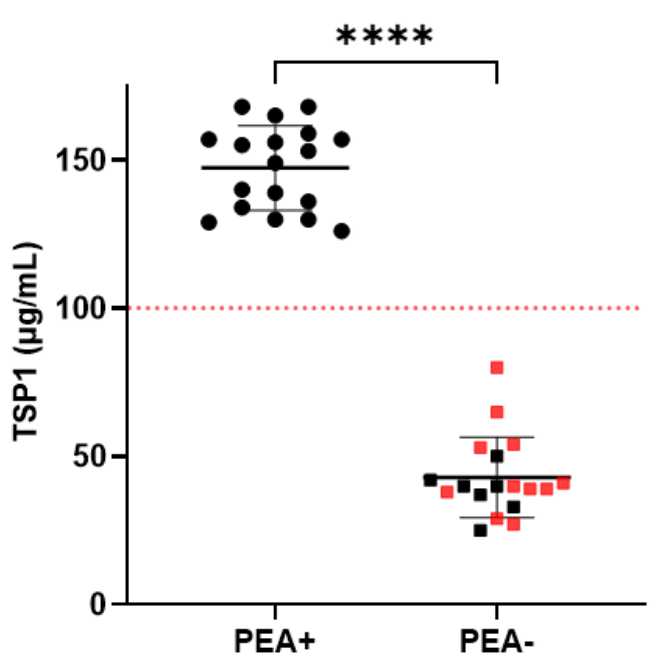

